Voix et Images

voixetimages

\title{
Bibliographie de Gilbert Langevin
}

\section{André Gervais}

Volume 22, numéro 3 (66), printemps 1997

\section{Gilbert Langevin}

URI : https://id.erudit.org/iderudit/201324ar

DOI : https://doi.org/10.7202/201324ar

Aller au sommaire du numéro

Éditeur(s)

Université du Québec à Montréal

ISSN

0318-9201 (imprimé)

1705-933X (numérique)

Découvrir la revue

Citer cet article

Gervais, A. (1997). Bibliographie de Gilbert Langevin. Voix et Images, 22(3),

520-543. https://doi.org/10.7202/201324ar d'utilisation que vous pouvez consulter en ligne.

https://apropos.erudit.org/fr/usagers/politique-dutilisation/ 


\section{Bibliographie de Gilbert Langevin}

\section{André Gervais, Université du Québec à Rimouski}

Trois remarques :

- Les entrées qui composent les sept sections de cette bibliographie/discographie/filmographie nullement définitive sont disposées chronologiquement.

- Il existe plusieurs autres bandes film ou vidéo (voir section IV), mais elles ne sont pas montées, ou encore le son (ou l'image) n'est pas tel (ou telle) qu'on l'aurait voulu; la recherche de ces "témoins" non officiellement "édités" ne fait que commencer.

- Les entrées relatives à la réception (voir section VI) mauricienne m'ont été fournies par Bernard Pozier, du cégep Joliette-De Lanaudière, celles relatives à la réception saguenayenne par Aurélien Boivin, de l'Université Laval, qui a aussi ajouté quelques détails à l'ensemble.

\section{LIVRES}

\section{A) Éditions courantes}

À la gueule du jour, Montréal, Éditions Atys, [mars] 1959, s.p. (repris dans Origines 1959-1967, 1971).

Symptômes, Montréal, Éditions Atys, [mars] 1963, s.p. (repris dans Origines 19591967, 1971).

Un peu plus d'ombre au dos de la falaise, Montréal, Éditions Estérel, [mai] 1966, 83 p. (repris dans Origines 1959-1967, 1971).

Pour une aube, Montréal, Éditions Estérel, [juillet] 1967, 72 p. (repris dans Origines 1959-1967, 1971).

Noctuaire, Montréal, Éditions Estérel, [juillet] 1967, 36 p. (repris dans Origines 1959-1967, 1971).

Stress, Montréal, Éditions du Jour, coll. "Les poètes du Jour ", [1 ${ }^{\text {er }}$ trimestre] 1971, $47 \mathrm{p}$.

Ouvrir le feu, Montréal, Éditions du Jour, coll. “Les poètes du Jour *, ljanvier] 1971, $60 \mathrm{p}$.

Origines 1959-1967, Montréal, Éditions du Jour, loctobre] 1971, 275 p.

Les écrits de Zéro Legel, première série, Montréal, Éditions du Jour, coll. "Prose du Jour ", [4 trimestre] 1972, $156 \mathrm{p}$.

Chansons et poèmes, Montréal, Éditions Vert blanc rouge/Éditions Québécoises, coll. "Poésie ", no 4, [juin ou juillet] 1973, 78 p. 
Novembre suivi de La vue du sang, Montréal, Éditions du Jour, coll. •Poètes du Jour , [août] 1973, 84 p.

La douche ou la seringue [Les écrits de Zéro Legel, deuxième série], postface de Lucien Francœur, Montréal, Éditions du Jour, coll. "Prose du Jour ", [décembre] 1973, $115 \mathrm{p}$.

Chansons et poèmes 2, Montréal, Éditions Vert blanc rouge/Éditions Québécoises, [juin] 1974, $76 \mathrm{p}$.

Griefs, poégrammes, Montréal, l'Hexagone, [décembre] 1975, 69 p.

L'avion rose. Écrits de Zéro Legel, troisième série, Montréal, Éditions La Presse, [1 ${ }^{\mathrm{er}}$ trimestre] $1976,102 \mathrm{p}$.

Mon refuge est un volcan, avec neuf illustrations de Carl Daoust, Montréal, l'Hexagone, [décembre] 1977, 93 p. (Sur la page "Du même auteur ", une section distincte s'ajoute : * Discographie sommaire •.)

Le fou solidaire, illustrations de Jocelyne Messier, Montréal, l'Hexagone, [juin] $1980,71 \mathrm{p}$.

Issue de secours, illustrations de l'auteur, Montréal, l'Hexagone, ljuillet] 1981, $76 \mathrm{p}$.

Les mains libres, Montréal, Éditions Parti pris, coll. •Paroles", $\mathrm{n}^{\circ}$ 62, [décembre] $1983,86 \mathrm{p}$.

Entre l'inerte et les clameurs, Trois-Rivières, Écrits des Forges, coll. * Radar *, $\mathrm{n}^{\circ} 15$, [mars] 1985, $51 \mathrm{p}$.

Comme un lexique des abîmes, Trois-Rivières, Écrits des Forges, coll. "Radar", $\mathrm{n}^{\circ} 19$, [mars] 1986, $70 \mathrm{p}$.

Au plaisir, Trois-Rivières, Écrits des Forges, coll. * Radar •, n 25, [septembre] 1987, $61 \mathrm{p}$.

La saison hantée, Trois-Rivières, Écrits des Forges, coll. * Radar *, n ${ }^{\circ} 32$, [septembre] $1988,79 \mathrm{p}$.

Ultimacolor suivi de Espace appelle écho, Jonquière, Éditions Sagamie/Québec, [septembre] 1988, $92 \mathrm{p}$.

Né en avril, textes poétiques suivis d'une lettre manuscrite de Gaston Miron, TroisRivières, Écrits des Forges, coll. *Radar *, no 37, [juillet] 1989, 62 p. (Sur la page "Du même auteur", une section distincte s'ajoute: "Filmographie et vidéographie m.)

Haut risque, Trois-Rivières, Écrits des Forges, coll. "Radar ", no 50, [août] 1990, $59 \mathrm{p}$.

Les vulnérables, Montréal, l'Hexagone, coll. •Poésie *, [décembre] 1990, 61 p. (écrit en collaboration avec Jean Hallal).

Le dernter nom de la terre, Montréal, l'Hexagone, coll. • Poésie •, [décembre] 1992, $87 \mathrm{p}$.

Confidences aux gens de l'archipel, quatrième série des écrits de Zéro Legel, Montréal, Éditions Triptyque, [juillet] 1993, 85 p.

Le cercle ouvert suivi de Hors les murs, Chemin fragile et L'eau souterraine, Montréal, l'Hexagone, coll. "Poésie , [août] 1993, 175 p. 
PoéVie. Poésie, chansons, prose et aphorismes, choix de textes et présentation par Normand Baillargeon, Montréal, Éditions Typo, 1997.

La voix que jai, chansons choisies, édition préparée par André Gervais, Montréal, VLB éditeur, coll. "Chansons et monologues", 1997.

\section{B) Hors commerce}

Le vertige de sourire; Montréal, Éditions Atys, [novembre] 1960, s.p. (Sur la page "Du même auteur ", de 1963 à 1967, l'éditeur est Atelier Pierre Guillaume ou, simplement, Guillaume.)

Poèmes à l'effigie de Larouche, Larsen, Miron, Carrier, Châtillon, Caron, Marguère et mot, Montréal, Éditions Atys, [novembre ou décembre] 1960, s.p. (Sur la page «Du même auteur ", à partir de 1966, ce titre est condensé en Poèmeseffigies.)

\section{C) Livres d'artistes}

Les imagiers, poème de G.L. («Au milieu de la nuit») illustré par douze eauxfortes, bois gravés ou linogravures de Kittie Bruneau, Daniq Charland, Sandra Cole, M. Françoise Condamin, Monique Dussault, Réjean Lemay, Janine Leroux-Guillaume, Anne Lewis, Mariel Pilon, Denis Schneider, Shagri et Josette Trépanier, préface de Françoise Bujold, composition et impression typographiques par Pierre Guillaume, boîtier de Pierre Ouvrard, Montréal, Éditions Sagitta, [mai] 1977, s.p. (repris, sans les illustrations, dans Mon refuge est un volcan, 1977).

Fables du temps rauque (pour l'enfant d'autrefots), poèmes de G.L. avec sept bois gravés de Monique Dussault, composition et impression typographiques par Pierre Guillaume, boîtier de Pierre Ouvrard, Montréal, Éditions du Pôle, [septembre] 1981, s.p. (repris, sans les illustrations, dans Les mains libres, 1983).

L'île, onze estampes et onze poèmes, liminaire de Guy Robert, boîtier de Pierre Ouvrard, Val-David, Atelier de l'Ile, et Ville Mont-Royal, Iconia, Guy Robert éditeur, coll. "Suite québécoise *, [mars] 1983, s.p.; à chaque graveur un auteur: Jocelyne Aird-Bélanger/Michèle Lalonde, Bonnie Baxter/Jean Hallal, Chantal Lévesque / Benoît Lacroix, Adriano Lambe/Yves-Gabriel Brunet, Louise Cloutier/Pierre Morency, Marcel Carrier/Réal Huot, Michel-Thomas Tremblay/Paul Chamberland, Gilles Boisvert/G.L. ("Vers une île en cœur"), Pierre Leblanc/Raoul Duguay, Jocelyne Benoît/Clémence DesRochers, et Francine Beauvais/Janou Saint-Denis (repris, sans l'illustration, dans Les mains libres, 1983).

\section{TEXTES, ÉCRITS ET PARLÉS PUBLIÉS SÉPARÉMENT, ENSUITE REPRIS (OU NON)'DANS LES LIVRES}

\section{A) Poèmes}

"La source ", dans $A u$ large, journal étudiant du Séminaire Saint-Alphonse, SainteAnne-de-Beaupré, février 1955, p. 13 (repris sous le titre de "Une source. dans L'Étoile du lac, Roberval, 24 mars 1955, p. 2). 
"Ivresse poétique ", dans L'Étotle du lac, Roberval, 5 mai 1955, p. 10.

"Balade du soir ", dans L'Étoile du lac, Roberval, 16 juin 1955, p. 3.

"Jours de pluie ", dans L'Étoile du lac, Roberval, 11 août 1955, p. 10.

"Nocturne ", dans $A u$ large, journal étudiant du Séminaire Saint-Alphonse, SainteAnne-de-Beaupré, mars 1956, p. 32 (repris dans Le Réveil, JonquièreKénogami, 22 mai 1957, p. 13).

Gyl BERGEVIN [pseud. de G.L.], "Estivales ", dans Nouveautés poétiques, Roberval, Éditions Atys, [juillet 1958], s.p.

Régis AUGER [pseud. de G.L.], "Québécaillerie ", Les Cabiers fraternalistes, Montréal, Éditions Atys, coll. "Silex", n 5, mars-avril 1964, p. $17-19$ (repris dans L'avion rose, 1976).

Zéro LEGEL [pseud. de G.L.], "Croc-en-jambes ", "Éloge succinct d'un premier ministre mort en temps de paix " et "Torpilles ", Les Cabiers fraternalistes, Montréal, Éditions Atys, coll. :Silex", no 5, mars-avril 1964, p. $20-22$ (repris dans Les écrits de Zéro Legel, 1972).

"La nuit profite...", "Dans la cohue..." et "Ton âme chemine... ", Passe-partout, Saint-Constant, vol. I, $\mathrm{n}^{\circ} 1$, janvier 1965, p. 13-15 (repris dans Un peu plus d'ombre au dos de la falaise, 1966).

Régis AUGER [pseud. de G.L.], "Le tonnerre apprivoisé " et "Show time ou L'invitation à l'amour ", Passe-partout, Saint-Constant, vol. I, n ${ }^{\circ} 4$, avril 1965, p. 12-13.

"Je verrouille..." et "Originent de la rue...", Passe-partout, Saint-Constant, vol. I, $\mathrm{n}^{\circ}$ 5, mai 1965, p. 8-9 (repris dans Un peu plus d'ombre au dos de la falaise, 1966).

“Noctuaire ", Passe-partout, Saint-Constant, vol. I, n 9 , septembre 1965, p. 2-3 (repris dans Noctuaire, 1967).

"Noctuaire II. Poégrammes ", Passe-partout, Saint-Constant, vol. I, n ${ }^{\text {os }}$ 11-12, novembre-décembre 1965, p. 4-15 (repris dans Noctuaire, 1967).

Zéro LEGEL [pseud. de G.L.]: "Parole de cible: ici lance ", La Barre du jour, Montréal, $\mathrm{n}^{\circ} 14$, juin-juillet 1968, p. 29-31 (repris dans L'avion rose, 1976).

"Corps fébrile que les semences émeuvent..." "Je suis un produit de votre fiasco..." et "Mes rêves ont des pensées de sable...", Les Herbes rouges, Montréal, no 2, décembre 1968-mars 1969, p. 7-9 (repris dans Stress, 1971).

"Je déchire ce qui reste pêle-mêle...", *Entrer par tes yeux...", "J'éprouve de nouveau le sommeil/difficulté... et . Nage homme dans le mesquin... *, Europe, Paris, $47^{\mathrm{e}}$ année, $\mathrm{n}^{\text {os }} 478-479$, février-mars 1969, p. 213-214 (repris dans Stress, 1971).

"Avec les simples munitions...", "Mots grêles..." et "Moi l'ouvert au délire...", Métamorphoses, Limoges, $\mathrm{n}^{\circ} \mathrm{IX}, 2^{\mathrm{e}}$ trimestre 1969, s.p. (repris dans Stress, 1971).

"Saison difficultueuse...", "Avec les simples munitions... " et "Moi l'ouvert au délire... *, dans Guy Robert, Littérature du Québec. Poésie actuelle, Montréal, Librairie Déom, 1970, p. 308-309 (repris dans Stress, 1971). 
"Pour ou contre l'Immaculée Contraception - et * Douche-épître aux Minustres ", Hobo-Québec, Québec, no 3; mars 1973, p. 3 (premier poème repris partiellement dans La douche ou la seringue, 1973, et partiellement dans Chansons et poèmes 2, 1974 ; second poème dans La doucbe ou la seringue, 1973).

"La moppe su'a mappe ", La Presse, Montréal, 21 juillet 1973, p. D3 (repris dans Chansons et poèmes 2, 1974).

"Où sont les étrennes de la paix promise?", Le Jour, Ville Saint-Laurent, 28 décembre 1974, p. 12 (repris dans L'avion rose, 1976).

"Le fou solidaire (extraits)., Hobo-Québec, Québec, $\mathrm{n}^{\text {os }} 25-26$, septembredécembre 1975, p. 4 (repris dans Mon refuge est un volcan, 1977).

"Remaniement sensoriel ", poème calligraphié sur beau papier à l'occasion de la campagne intitulée " 100,000 prétextes pour danser, des Grands Ballets canadiens ", Montréal, octobre 1975 (?)-mars 1976 (repris dans L'avion rose, 1976).

*Une île entre mille (extraits) ", Hobo-Québec, Québec, nos 27-28, janvier-avril 1976, p. 6 (repris dans Mon refuge est un volcan, 1977).

"Occultez-nous pour voir", Hobo-Québec, Québec, n ${ }^{\text {os }} 29-30$, mai-août 1976, p. 8 (repris dans Mon refuge est un volcan, 1977).

"Mon refuge est un volcan/extraits", Hobo-Québec, Québec, no 31, septembredécembre 1976, p. 9 (repris dans Mon refuge est un volcan, 1977).

"L'innocence en ruine/extraits ", Hobo-Québec, Québec, n’ 32, janvier-mars 1977, p. 10 (repris dans Mon refuge est un volcan, 1977).

"Quand la vie forge..." et "La fête coule... ", Focus, Jonquière, vol. I, n 4, septembre 1977, p. 25 (repris dans Mon refuge est un volcan, 1977).

"Au milieu de la nuit et Autres indices", Estuatre, Québec, no 6, décembre 1977, p. 87-93 (repris dans Mon refuge est un volcan, 1977).

"Le fou solidaire ", Liberté, Montréal, no 120 , novembre-décembre 1978, p. 71-72 (repris dans Le fou solidatre, 1980).

Zéro LEGEL alias Gilbert LANGEVIN dit LACROIX: "La revanche des sleepingbags ", Hobo-Québec, Québec, $n^{\text {os }} 36-37$, janvier-mars 1979, p. 5 (repris dans Le fou solidaire, 1980).

"Pour un sillon fertile... ", Hobo-Québec, Québec, nos 38-39, automne 1979, p. 48 (repris dans Le fou solidaire, 1980).

"Ixe-temps ", Hobo-Québec, Québec, n 40, hiver 1979-1980, p. 11 (repris dans Le fou solidaire, 1980).

Zéro LEGEL [pseud. de G.L.]: "Nous entrons dans l'ère de l'Ovnir... ", HoboQuébec, Québec, nº 40, hiver 1979-1980, p. 11.

-Le fou solidaire (extraits)., Hobo-Québec, Québec, no 41-42, été 1980, p. 45 (repris dans Le fou solidaire, 1980).

"Fever forever ", Hobo-Québec, Québec, no 43, hiver 1980-1981, p. 28 (repris dans Issue de secours, 1981). 
"Aimer est un très grand pays...", Hobo-Québec, Québec, n ${ }^{\text {os }} 46-47$, automnehiver 1981, p. 8 (repris dans Les mains libres, 1983).

"Boire le ciel/boire l'orage ", avec une illustration de Monique Dussault, Résistances, Jonquière, $\mathrm{n}^{\mathrm{o}} 1$, hiver 1982, p. 3-7 (les deux premiers poèmes repris dans Les mains libres, 1983).

Zéro LEGEL [pseud. de G.L.], "Self-defence ", accompagné de dessins de Gilbert Langevin, Terminus, Montréal, $\mathrm{n}^{\circ} 8,1986$, p. 2.

"Ainsi que rebelle (fragments)", accompagné de dessins de Gilbert Langevin, Terminus, Montréal, $\mathrm{n}^{\circ}$ 8, 1986, p. 3 (repris dans Au plaisir, 1987).

"Au vent neuf ", Kérosène, Montréal, septembre (?) 1986, p. 4-5 (repris dans $A u$ plaisir, 1987).

"Ouananiche", s.l.n.d., poème publié sous deux formes (dépliant et affiche) par les Éditions Sagamie/Québec, Jonquière, à l'occasion de l'hommage à Gilbert Langevin fait par le $20^{\mathrm{e}}$ Salon du livre du Saguenay-Lac-Saint-Jean, Jonquière, 29 septembre-2 octobre 1988 (repris dans Haut risque, 1990).

"D'un oiseau à l'autre", accompagné de dessins de Zéro Legel, Il y $a$, Montréal, n 3 , décembre 1993, p. 1 et 3.

"À Cybèle", Quatre. Cabier d'écriture (publié par La Société des poètes de la rue), Montréal, vol. 1, n 3, et vol. 2, no 1, décembre 1993 et janvier 1994, p. 56.

"Sans frontière" et "Eaux d'elle *, Résolu (publication photocopiée), Montréal, $\mathrm{n}^{\circ} 2$, [27 novembre 1995], p. 2-3.

\section{B) Paroles de chansons}

"Que finisse le temps des victimes... ("Le temps des vivants"), Le Voyage, Montréal, vol. I, n² 2, juin 1968, p. 4 (repris dans Chansons et poèmes, 1973).

"Le temps des vivants" et "J'entrevois", dans Guy Robert, Littérature du Québec. Poésie actuelle, Montréal, Librairie Déom, 1970, p. 309-312 (repris dans Chansons et poèmes, 1973).

"Le temps des vivants", dans Poèmes et chants de la résistance 2, cahier accompagnant le disque collectif, Montréal, 1971 (repris dans Chansons et poèmes, 1973).

"La prison des habitudes " et "La vie en bouteille", dans Gilbert Langevin, Body of Night, choix de poèmes et de paroles de chansons présentés et traduits par Marc Plourde, édition bilingue, Montréal, Éditions Guernica, coll. "Essential Poets ", $\mathrm{n}^{\mathrm{o}} 20$, [août] 1987, p. 90-95.

"Le vent bleu ", "Naufrage ", "Arvida-girl ", "La bartendresse * et "Suzanne ", Mobius, Montréal, $\mathrm{n}^{\circ}$ 40, printemps 1989, p. 117-123.

"Ange Animal", dans Heinz Weinmann et Roger Chamberland (dir.), Littérature québécotse, des origines à nos jours. Textes et métbode, Ville LaSalle, Hurtubise HMH, 1996, p. 318-319.

\section{C) Proses}

Gyl BERGEVIN [pseud. de G.L.], "Georges Larouche, sa vie et son œuvre ”, dans Nouveautés poétiques, Roberval, Éditions Atys, [juillet 1958], s.p. 
"Doréa. Royaume des recherches en déficience mentale ", Points de vue, SaintJérôme, février 1961, p. 12.

"malgré son jeune âge... *, dans Claude Laurier, D'un monstre à l'autre, Montréal, Éditions Atys, [novembre] 1961, $4^{\mathrm{e}}$ de couverture.

LE NOCTAMBULE [pseud. de G.L.], *Auto-questionnaire (le fraternalisme au service de l'évolution) ", Les Cabiers fraternalistes, Montréal, Éditions Atys, coll. *Silex *, n ${ }^{\circ}$ 5, mars-avril 1964, p. 23-24.

Zéro LEGEL [pseud. de G.L.], • Flash-back •, Quoi, Montréal, vol. I, no 1, janvierfévrier 1967, p. 39-52 (repris dans Les écrits de Zéro Legel, 1972).

Alfred DESROCHERS, Jacques GODBOUT, Gilbert LANGEVIN, Claude PÉLOQUIN, André MAJOR et Réjean DUCHARME, -Témoignages d'écrivains " (sur Émile Nelligan), Études françaises, Montréal, vol. III, no 3, août 1967 (celui de Gilbert Langevin se trouve à la p. 304).

„Poé-vie? •, dans Guy Robert, Littérature du Québec. Poésie actuelle, Montréal, Déom, 1970, p. 299.

"Interdire le port de langue - (lettre à Réginald Martel, $1^{\mathrm{er}}$ juillet 1973), La Presse, Montréal, 21 juillet 1973, p. D3.

«Pour ou contre Straram? ", Hobo-Québec, Québec, n 9, octobre-novembre 1973, p. 5.

Zéro LEGEL [pseud. de G.L.], *Trois istoires en forme de $\mathrm{H}$ ", accompagnées de dessins de Zéro Legel, Hobo-Québec, Québec, $\mathrm{n}^{\text {os }} 14-15$, janvier 1974, p. 10 (repris dans L'avion rose, 1976).

"19- pour un nouvel [sic] Montréal ", Le Devoir, Montréal, 18 mai 1974, p. XXVI.

"Vaincrons-nous? Continuons le combat *, Maintenant, Montréal, n ${ }^{\mathrm{os}} 137-138$, juinseptembre 1974 , p. 34.

Gilbert LANGEVIN et Alain TITTLEY, * Reproches de poète [...] * (lettre à Philippe Haeck), Le Devoir, Montréal, 10 mai 1975, p. 24.

"Contre-culture ou réforme grégaire?", Hobo-Québec, Québec, n ${ }^{\text {os }}$ 25-26, septembre-décembre 1975, p. 4.

"Point de vue fraternaliste•, Hobo-Québec, Québec, n ${ }^{\text {os }}$ 27-28, janvier-avril 1976, p. 6 (repris dans L'avion rose, 1976).

"À propos de L'avion rose ", La Presse, Montréal, 8 mai 1976, p. D3, et Le Devoir, 8 mai 1976, p. 17.

Zéro LEGEL [pseud. de G.L.], “Notes subjectales ou bibiographiques [sic] ", HoboQuébec, Québec, n ${ }^{\text {os }} 29-30$, mai-août 1976, p. 8 (repris dans Confidences aux gens de l'archipel, 1993).

"L'écrivain appartient d'abord à son peuple . (lettre à Roger Lemelin, 10 août 1976), Le Devoir, Montréal, 13 août 1976, p. 4.

"Lettre ouverte aux esprits fermés ", Hobo-Québec, Québec, n 31, septembredécembre 1976, p. 8.

"Ả la mémoire de Gaby Johnston (21 septembre 1938-17 novembre 1976) ", HoboQuébec, Québec, n 32, janvier-mars 1977, p. 10. 
"Journal hors-bord/chronique athéorique (1) ", Hobo-Québec, Québec, $n^{\circ} 33$, avriljuin 1977, p. 6.

"Préface ", dans Janou Saint-Denis, Claude Gauvreau le cygne, Montréal, Presses de l'Université du Québec, 1978, p. 11-12.

"Déclaration de Gilbert Langevin " (lors de la remise du prix du Gouverneur général du Canada, 4 avril 1979), Le Devoir, Montréal, 21 avril 1979, p. 23 (repris sous le titre de "Déclaration à Rideau Hall/4 avril 1979", Hobo-Québec, Québec, $\mathrm{n}^{\text {os }} 38-39$, automne 1979, p. 48).

"Georges Larouche ou l'extase baroque ", Résistances, Jonquière, $\mathrm{n}^{\circ} 2$, étéautomne 1982, p. 41-43.

"Il y a chez Morisseau... (28 août 1987), dans Roland Morisseau, Poésie (19601991), Montréal, Éditions Guernica, coll. "Voix ", n 23, 1993, $4^{\mathrm{e}}$ de couverture.

\section{D) Parlés}

Madeleine LEBLANC, "Propos d'un jeune éditeur ", Le Droit, Ottawa, 24 novembre 1962 , p. 8.

Gilles MARCOTTE, [entrevue avec Gilbert Langevin] (à propos de Noctuaire, 1967, et de Pour une aube, 1967), série Des livres et des bommes, Gilbert Picard réalisateur, CBF-FM, 27 février 1968.

ANONYME, "Quatre poètes et la poésie * (entrevues avec Roland Giguère, Gilbert Langevin, Pierre Morency et Fernand Ouellette), Culture vivante, Montréal, $\mathrm{n}^{\circ} 12$, février 1969 , p. 6-11.

Jean ROYER, “L'ange XX comme dans siècle ", L'Action, Québec, 25 octobre 1969, p. 15.

Jean SARRAZIN, [Entrevue avec Gilbert Langevin] (à propos d'Ouvrir le feu, 1971, et de Stress, 1971), série Carnet arts et lettres, Gilbert Picard réalisateur, CBF-FM, 3 février 1971.

Réginald MARTEL et Jacques GODBOUT, [entrevue avec Gilbert Langevin] (à propos d'Ouvrir le feu, 1971, et de Stress, 1971), série Horizons, Gilles Archambault réalisateur, CBF-FM, 4 février 1971.

Michel BEAULIEU, [entrevue avec Gilbert Langevin] (à propos de Rina Lasnier), série L'atelier, Aline Legrand réalisatrice, CBF-FM, 25 mai 1971.

Réginald MARTEL et Jacques GODBOUT, [entrevue avec Gilbert Langevin] (à propos d'Origines 1959-1967, 1971), série Book-club, Gilles Archambault réalisateur, CBF-FM, 7 février 1972.

Jean SARRAZIN, [entrevue avec Gilbert Langevin] (à propos des Écrits de Zéro Legel, 1972), série Carnet arts et lettres, Raymond Fafard réalisateur, 'CBF-FM, 7 février 1973.

François HÉBERT, Marcel HÉBERT et Claude ROBITAILLE, "Interview: Gilbert Langevin ", Hobo-Québec, Québec, nos 5-7, juin-août 1973, p. 22-23 et 26-27.

Jeannine PAQUET, [entretien avec Gilbert Langevin], série Leur violon d'Ingres, Pierre Rainville réalisateur, CBF-FM, 3 novembre 1973. 
Jean-Claude TRAIT, "Langevin, poète à la fois cactus et fleur bleue ", La Presse, Montréal, 19 janvier 1974, p. D2.

Gaëtan DOSTIE, "Lancement de Chansons et poèmes 2. Gilbert Langevin, messager de l'événement •, Le Jour, Ville Saint-Laurent, 6 juillet 1974, p. V2.

"Gilbert Langevin et le Lac-Saint-Jean", Jean Doyon lecteur, série Un écrivain et son pays, Jean Lacroix réalisateur, CBF-FM, 3 mars 1975.

Yvon PARÉ, *Gilbert Langevin, un vrai bleuet de la ville•, Progrès-Dimanche, Chicoutimi, $1^{\text {er }}$ juin 1975 , p. 2 B.

Jocelyn PAGÉ, *Entrevue avec Gilbert Langevin *, Focus, Jonquière, vol. I, no 4 , septembre 1977 , p. $20-25$.

Jean ROYER, "Gilbert Langevin: la vie d'un autre *, Le Devoir, Montréal, 12 août 1978 , p. 13 (repris, par exemple, dans Jean Royer, Poètes québécots. Entretiens, Montréal, l'Hexagone, coll. "Typo", 1991, p. 143-148).

Yvon PARÉ, "Sans les mots, il n'y a pas de vie. Gilbert Langevin, poète avant tout ", Le Quotidien, Chicoutimi, 19 mai 1979, p. 2-3.

Jean-Guy MARTIN, "Il vaut mieux suivre la flèche que la recevoir" - Gilbert Langevin ., Journal de Montréal, Montréal, $1^{\mathrm{er}}$ novembre 1980, p. 5.

[Pierre LABELLE], [Propos de Gilbert Langevin], dans Gilbert Langevin, Où mes racines parlent d'elles-mêmes (face A) et Le fou soltdaire (face B), entrevue, poèmes et chansons, 33 tours, présentation de [Jacques Brault], Pierre Labelle réalisateur, Montréal, Radio-Canada International, F-774, 1980.

Antonio D'ALFONSO, «Le fou solidatre, poèmes de Gilbert Langevin, le livre du mois choisi par le comité de rédaction (voir n. 31). Gilbert Langevin répond aux questions de notre collaborateur, Antonio D'Alfonso ", Nos livres, Montréal, vol. XIII, janvier 1982, s.p.

Normand BAILLARGEON, "La parole optimiste. L'infatigable parleur perçoit toujours les mots comme des choses ", Le Devoir, Montréal, 14 février 1994, p. B1.

\section{DISCOGRAPHIE}

\section{A) Chansons}

"Comme je crie/comme je chante. (musique: François Cousineau)

"Il y a " (musique: François Cousineau)

"Le baraubord "(musique: François Cousineau)

- La vie continue. (musique: G.L.)

"Exil. (musique: Jean Fortier)

«Urba * (musique: Jacques Perron)

"J'entrevois" (musique: François Cousineau)

"La solitude encore " (musique: François Cousineau)

"Les chasseurs de pucelles " (musique: François Cousineau)

"Suzanne (paroles et musique : Leonard Cohen, adaptation française : G.L.)

"Le temps des vivants . (musique: François Cousineau)

par Pauline JULIEN, Comme je crie... comme je chante..., 33 tours, Montréal, Gamma, GS 125, 1969; les enregistrements de "Comme je crie/comme je 
chante ", "Suzanne * et "Le temps des vivants * sont repris dans Pauline Julien, Québec Love. La collection, CD, Montréal, Gamma, GCD-505, 1993.

"Le temps des vivants * (musique: François Cousineau) (version inédite) par Pauline JULIEN, dans un disque collectif, Poèmes et chants de la résistance 2, album double enregistré en public (au Gésù, Montréal, 24 janvier 1971), Montréal, Résistance, RE 604, 1971.

"À la dérive "(musique: Richard Grégoire)

"Dans l'temps comme dans l'temps" (musique: Jacques Perron) par Pauline JULIEN, Licence complète, 33 tours, Montréal, Zodiaque, ZOX 6018, 1974.

"La voix que j'ai " (musique: Jean Gravel et Gérald Boulet) par OFFENBACH, Offenbach, 33 tours, Montréal, A\&M 9027, 1977.

"Cette voix * (fragment récité de * La voix que j'ai *)

"D'où que vienne" (musique: G.L.)

"Blanche douceur " (musique: G.L.)

"Naturel. (musique: G.L.)

"Il y a des autos (musique: G.L.)

"Quand on fait de la peine * (musique: G.L.)

“On est là - (musique: G.L.)

"Le temps des vivants" (récité) par Gilbert LANGEVIN, Où mes racines parlent d'elles-mêmes/Le fou solidaire, 33 tours, Montréal, Radio-Canada International, F-774, 1980.

"L'étoile du nord * (musique: Claude Gauthier) par Pauline JULIEN, Charade, 33 tours, Montréal, Kébec-Disc, KD-535, 1982.

"Celle qui va * (musique: Pascal Mailloux et Jacques Noël) par MARJO, Celle qui va, 33 tours, Montréal, Kébec-Disc, KD-651, 1986.

"Naufrage" (musique: Dan Bigras) par Dan BIGRAS, disque simple, Montréal, GMD 1301-14, 1988.

"La voix que j'ai * (musique: Jean Gravel et Gérald Boulet) (version inédite) par OFFENBACH, Offenbach/C'était plus qu'une aventure 1972 à 1985, CD, Montréal, Disques Double, CD-30004, 1989.

"Ange Animal " (musique: Dan Bigras) par Dan BIGRAS, Ange Animal, CD, Montréal, GMD 1303-30, 1990; l'enregistrement de "Naufrage " est repris sur ce disque.

"Hôtel Vert-de-Gris " (musique: Daniel Marcoux) par Yves RAYMOND, Yves Raymond, CD, Montréal, Eschyle, ES DI 111 10-01, 1991.

"Le vent bleu * (musique: Dan Bigras)

par Dan BIGRAS, Tue-mot, CD, Montréal, Leila, CPLCD-60, 1992.

"Le blé des autres - (musique: Jean Custeau)

"Ruelle de nos amours " (musique: Jean Custeau)

"Arthur " (musique: Jean Custeau)

"Entre un scotch et du jazz - (musique: Jean Custeau) par Jean CUSTEAU, Custeau, CD, Magog, VAMMA, VMA 8348-2, 1992. 
"Le train maudit " (musique: Stephen Faulkner)

par Stephen FAULKNER, Caboose, CD, Montréal, Faucon blanc, FB 1001-2, 1992.

"Si ciel il y a * (musique: G.L.)

par Pierre FLYNN, dans un disque collectif, $A$ u nom de l'amour, CD, Montréal, ANL, CD 666 2, 1993.

"Naufrage * (musique: Dan Bigras) (version inédite)

"Ange Animal * (musique: Dan Bigras) (version inédite)

par Dan BIGRAS, Le fou du diable, CD, Montréal, DAACD-9200, 1995.

"Dans le cri de nos nuits. (musique: Dan Bigras)

par Luce DUFAULT, Luce Dufault, CD, Montréal, Arpège Musique, AMCD 904, 1996.

"D'où que vienne. (musique: G.L.)

"Blanche douceur" (musique: G.L.)

"Fous solidaires (complainte). (musique: Dominique Tremblay)

*Naturel - (musique: G.L. - reprise de la bande 1980)

"Si ciel il y a* (musique: G.L.)

"D'un cœur à l'autre : (musique: Dominique Tremblay)

"Le mur" (musique: G.L.)

"Aimer (musique: Dominique Tremblay)

par Hélène TREMBLAY (voix) et Dominique TREMBLAY (violon), Langevin/ Tremblay/Fous solidaires, CD, Val-Morin, SRM, SRM 02502, 1996.

\section{B) Poèmes}

"Je bénissais ton ventre. (tiré de Pour une aube, 1967)

"J'ivre meurs " (tiré de Chansons et poèmes, 1973)

"Mirage le ventre * (tiré de Le fou solidaire, 1980)

"Chaque matin " (tiré de Un peu plus d'ombre au dos de la falaise, 1966)

"Je reviendrai * (tiré de Le fou solidaire, 1980)

par Gilbert LANGEVIN, Où mes racines parlent d'elles-mêmes/Le fou solidaire, 33 tours, Montréal, Radio-Canada International, F-774, 1980.

"Généalogie du futur " (tiré de Symptômes, 1963)

"Funérailles " (tiré de Symptômes, 1963)

"Naissance. (tiré de Symptômes, 1963)

"Face à demain " (tiré de $A$ la gueule du jour, 1959)

"Le blé des autres ou Le diseur de mésaventure. (tiré de Les écrits de Zéro Legel, 1972)

par Jean-Marie DA SILVA, 13 escales au pays. 33 paysages poétiques, 33 tours, Sainte-Sophie, Studio-Théâtre da Silva, 1989.

\section{FILMOGRAPHIE}

Jean-Louis FRUND, Jean-Gauguet [sic] Laroucbe sculpteur, court métrage documentaire, Québec, Ciné Québec (distributeur), 1965; commentaire de G.L.

Jean BISSONNETTE, Dimanshowsoir, émission de télévision, Montréal, RadioCanada, 6 février 1977; "Ce soir j'ai l'âme à la tendresse avec Pauline Julien. 
(enregistré le 5 février 1977) présente les écrivains Jean-Claude Germain, G.L., Gaston Miron et Michel Tremblay, ainsi que les pianistes Gaston Brisson et Jacques Perron.

Jean-Claude LABRECQUE et Jean-Pierre MASSE, La nuit de la poésie. 28 mars 1980, long métrage documentaire, Montréal, ONF, 1980; extraits du spectacle animé par Michel Garneau et Janou Saint-Denis à l'Université du Québec à Montréal (enregistré durant la nuit du 28 au 29 mars 1980).

Jean-Claude LABRECQUE, Paroles du Québec. Les sept paroles du Québec, émission de télévision, Montréal, Radio-Québec [aujourd'hui: Télé-Québec], 9 décembre 1981, dans le cadre de l'Émission spéciale du mercredi; extraits du spectacle (enregistré le 6 juillet 1980) d'Yves-Gabriel Brunet, Paul Chamberland, Raoul Duguay, Michel Garneau, Michèle Lalonde, G.L. et Gaston Miron, accompagnés par sept musiciens dirigés par le violoniste Dominique Tremblay, à la Maison de la culture de La Rochelle (France).

\section{$\checkmark$ TRADUCTIONS}

- en anglais:

"Poems by Gilbert Langevin ", vingt poèmes traduits par Barbara Belyea, Jay Bochner, Susan Copeland, Judith Cowan, Sheila Fischman, D.G. Jones, Ben-Z. Shek, Larry Shouldice, Tom Tebbutt et Mary Van Wyck, édition bilingue, Ellipse, Sherbrooke, $\mathrm{n}^{\circ} 13,1973$, p. 8-31.

Body of Night, choix de poèmes et de paroles de chansons présentés et traduits par Marc Plourde, édition bilingue, Montréal, Éditions Guernica, coll. "Essential Poets", $\mathrm{n}^{\circ}$ 20, [août] 1987, 138 p. (Certaines de ces traductions ont été publiées dans Rubicon, Montréal, $\mathrm{n}^{\circ}$ 3, dans une section intitulée "Seven Quebec Poets in Translation *, été 1984, p. 118-125.)

- en italien:

"Fables du temps rauque (extraits)", dans Jean Yves Collette/Nicole Deschamps, Brise-lames. Antbologie de la poésie moderne du Québec/Antemurale. Antologia della poesia moderna dal Québec, extraits traduits par Gennaro Pessini, édition bilingue, Rome, Bulzoni, 1990, p. 201-205.

\section{RÉCEPTION CRITIQUE}

\section{A) Études}

DUGUAY, Raoul, “3. Gilbert Langevin, poète maudit ", Parti pris, Montréal, vol. IV, $\mathrm{n}^{\circ} 1$, septembre-octobre 1966, p. 97-101.

PAGEAU, René, "Poésie de Gilbert Langevin *, Culture, Québec, vol. XXVIII, n 1 , mars 1967, p. 32-37.

PARADIS, Suzanne, "Un regard de lanterne folle dans le désert des ombres", Le Soleil, Québec, 29 novembre 1969, p. 46.

MARCOTTE, Gilles, Le temps des poètes. Description critique de la poésie actuelle au Canada français, Montréal, HMH, 1969, p. 200-203.

BEAULIEU, Michel, "Treize ans de malentendu autour de Gilbert Langevin *, Presqu'Amérique, Québec, vol. I, n 7, mai-juin 1972, p. 34-35. 
NEPVEU, Pierre, "Gilbert Langevin, l'énergumène ", Études françaises, Montréal, vol. $9, \mathrm{n}^{\circ} 4$, novembre 1973 , p. $337-344$ (repris, traduit en anglais par Jean Vigneault, dans Ellipse, Sherbrooke, $\mathrm{n}^{\circ} 13,1973$, p. 32-41).

NEPVEU, Pierre, "La poétique de Gilbert Langevin ", Livres et auteurs québécois 1973, Québec, Presses de l'Université Laval, 1974, p. 312-324.

BOURASSA, André-G., Surréalisme et littérature québécoise, Montréal, Éditions l'Étincelle, 1977, p. 257-260; Surréalisme et littérature québécoise. Histoire d'une révolution culturelle, édition revue et augmentée, Montréal, l'Hexagone, coll. “Typo *, nº 8, 1986, p. 449-454.

[BRAULT, Jacques], [Présentation], dans Gilbert Langevin, Où mes racines parlent d'elles-mêmes/Le fou solidaire, 33 tours, Montréal, Radio-Canada International, F-774, 1980.

LEMAIRE, Michel, "Gilbert Langevin: la tête du poète ", Lettres québécoises, Montréal, $\mathrm{n}^{\circ} 24$, hiver 1981-1982, p. 36-38.

NEPVEU, Pierre, "À la gueule du jour, recueil de poésies de Gilbert Langevin ", dans Maurice Lemire (dir.), Dictionnaire des œuvres littéraires du Québec, tome III (1940-1959), Montréal, Éditions Fides, 1982, p. 28-30.

BORDELEAU, Francine, "Poèmes à l'effigie de Larouche, Larsen, Miron, Carrier, Châtillon, Caron, Marguère et moi et autres recueils [Pour une aube et Noctuaire] de Gilbert Langevin ", dans Maurice Lemire (dir.), Dictionnaire des œuvres littéraires du Québec, tome IV (1960-1969), Montréal, Éditions Fides, 1984, p. 691-693.

DICKSON, Robert, *Symptômes et Un peu plus d'ombre au dos de la falaise, recueils de poésies de Gilbert Langevin ", dans Maurice Lemire (dir), Dictionnaire des auvres littéraires du Québec, tome IV (1960-1969), Montréal, Éditions Fides, 1984, p. 846-848.

NEPVEU, Pière, *L'Hexagone et les nouveaux courants *, dans René Dionne (dir.), Le Québécois et sa littérature, Sherbrooke/Paris, Éditions Naaman/Agence de coopération culturelle et technique, 1984, p. 206-208.

NEPVEU, Pierre, "Gilchrist Langenoir ", dans L'écologie du réel. Mort et naissance de la littérature québécoise contemporaine, Montréal, Boréal, coll. • Papiers collés *, 1986, p. 117-125.

CLERC, Isabelle, "Chansons et poèmes, recueils de Gilbert Langevin ", dans Maurice Lemire (dir.), Dictionnaire des auvres littéraires du Québec, tome V (1970-1975), Montréal, Éditions Fides, 1987, p. 132-133.

DÉRY, Pierre-Justin et Ronald MILO, * Stress et autres recueils de poésies [Ouvrir le feu, Les écrits de Zéro Legel, Novembre suivi de La vue du sang, La douche ou la seringue et Griefs] de Gilbert Langevin , dans Maurice Lemire (dir.), Dictionnaire des cuures littéraires du Québec, tome V (1970-1975), Montréal, Éditions Fides, 1987, p. 847-851.

MARQUIS, André, a Le rêve fraternaliste. Les Éditions Atys ", dans Groupe de recherche sur l'édition littéraire au Québec (GRÉLQ), L'édition de poésie. Les Éditions Erta, Orphée, Nocturne, Quartz, Atys et l'Hexagone, entrevues et études rassemblées et présentées par Richard Giguère avec la collaboration d'André Marquis, Sherbrooke, Éditions Ex Libris, 1989, p. 163-190. 
DES ROCHES, François, "L'avion rose et autres recueils de poésies [Mon refuge est un volcan et Le fou solidaire] de Gilbert Langevin *, dans Gilles Dorion (dir.), Dictionnaire des cuvres littéraires du Québec, tome VI (1976-1980), Montréal, Éditions Fides, 1994, p. 66-68.

GERVAIS, André, "À propos d'une chanson "de" Gerry Boulet ", dans Sas, Montréal, Éditions Triptyque, 1994, p. 215-223.

GERVAIS, André, “Part visible de l'“œuvre-vie" ", La Presse, Montréal, 22 octobre 1995 , p. B7.

NEPVEU, Pierre, "Le vulnérable impétueux ", La Presse, Montréal, 22 octobre 1995, p. B7.

BAILLARGEON, Normand, "Gilbert Langevin. Comme un frère humain ", Le Devoir, Montréal, 28 octobre 1995, p. D1 et D2 (repris dans PoéVie, 1997).

\section{B) Comptes rendus des recueils}

\section{À la gueule du jour}

ROBILLARD, Jean-Paul, «Poésie et rimaillages... Dans la dernière fournée, il n'y a que quatre bons poètes ", Le Petit Journal, Montréal, semaine du 17 au 24 mai 1959, p. 111.

\section{Symptômes}

BASTIEN, André, "Livres récents de jeune poésie ", Le Petit Journal, Montréal, 21 juin 1964, p. A39.

SYLVESTRE, Guy, [sans titre], The University of Toronto Quarterly, Toronto, vol. XXXIII, n' 4 , juillet 1964, p. 503.

VACHON, André-F. [pseud. de G.-André Vachon], * Six poètes à la recherche d'euxmêmes ", Livres et auteurs canadiens 1963, Montréal, Éditions Jumonville, 1964 , p. 56.

\section{Un peu plus d'ombre au dos de la falaise}

THÉBERGE, Jean-Yves, *Après les Éditions Atys *, Le Canada français, Saint-Jean d'Iberville, 16 juin 1966, p. 42.

MAJOR, André, "CEuvres de cinq poètes canadiens ", Le Petit Journal, Montréal, 19 juin 1966, p. 39-40 (repris partiellement dans Livres et auteurs canadiens 1966, Montréal, Éditions Jumonville, 1967, p. 74).

PIAZZA, François, [sans titre], Échos-Vedettes, Montréal, 9 juillet 1966, p. 22.

\section{Pour une aube}

MORIN, Yvon, "Lectures d'hiver. Pour une aube "L'Évangéline, Moncton, 17 février 1968, p. 4.

PILON, Jean-Guy, "Trois recueils et deux bons poètes. Pierre Morency et Gilbert Langevin , Le Devoir, Montréal, 2 mars 1968, p. 12 (repris partiellement dans Livres et auteurs canadiens 1968, Montréal, Éditions Jumonville, 1969, p. 101102). 
THÉBERGE, Jean-Yves, “Les deux derniers Gilbert Langevin ", Le Canada français, Saint-Jean d'Iberville, 7 mars 1968, p. 26.

ANONYME, [sans titre], Ficbes bibliographiques de littérature canadienne, Montréal, Éditions Fides, no 433, mai 1968, s.p.

PLAZZA, François, "À la bonne page. Pour une aube et Noctuaire ", Échos-Vedettes, Montréal, 4 mai 1968, p. 19.

PONTAUT, Alain, "Un poète, un roman, une revue ", La Presse, Montréal, 22 juin 1968, p. 29.

SYLVESTRE, Guy, [sans titre], The University of Toronto Quarterly, Toronto, vol. XXXVII, n $\mathrm{n}^{\circ}$, juillet 1968, p. 586.

\section{Noctuaire}

PILON, Jean-Guy, *Trois recueils et deux bons poètes. Pierre Morency et Gilbert Langevin , Le Devotr, Montréal, 2 mars 1968, p. 12 (repris partiellement dans Livres et auteurs canadiens 1968, Montréal, Éditions Jumonville, 1969, p. 101102).

THÉBERGE, Jean-Yves, "Les deux derniers Gilbert Langevin ", Le Canada français, Saint-Jean d'Iberville, 7 mars 1968, p. 26.

ANONYME, [sans titre], Fiches bibliographiques de littérature canadienne, Montréal, Éditions Fides, $n^{\circ} 432$, mai 1968, s.p.

PIAZZA, François, "À la bonne page. Pour une aube et Noctuaire ", Échos-Vedettes, Montréal, 4 mai 1968, p. 19.

\section{Stress}

TREMBLAY, Denis, “Ouvrir le feu et Stress", Montréal-Matin, Montréal, 15 février 1971, p. 26.

MARTEL, Réginald, "La saison des poètes I ", La Presse, Montréal, 10 avril 1971, p. D3.

BEAUCHAMP, Germain, «Dépossession et vie •, Le Devoir, Montréal, 21 avril 1971, p. 8.

THÉBERGE, Jean-Yves, * Gilbert Langevin, 2 fois ", Le Canada français, Saint-Jean d'Iberville, 12 mai 1971, p. 68.

PARADIS, Suzanne, * Langevin et Saint-Pierre. Demeurant à l'abri de toute définition ", Le Soleil, Québec, 31 juillet 1971, p. 34.

ANONYME, [sans titre], Le Livre canadien, Montréal, vol. II, 1971, n² 217, s.p.

MARCOTTE, Gilles, “La poésie - Pour l'âme *, Études françaises, Montréal, vol. VIII, $\mathrm{n}^{\circ} 1$, février 1972, p. 94-95.

DIONNE, René, *Sur les voies de notre poésie - IV *, Relations, Montréal, no 371 , mai 1972, p. 157-158.

MAILHOT, Laurent, [sans titre], Livres et auteurs québécois 1971, Montréal, Éditions Jumonville, 1972, p. 138-139. 


\section{Ouvrir le feu}

TREMBLAY, Denis, : Ouvrir le feu et Stress •, Montréal-Matin, Montréal, 15 février 1971, p. 26.

ANONYME, "Gilbert Langevin: Ouvrir le feu ", L'Action, Québec, 20 février 1971, p. 19.

MARTEL, Réginald, "La saison des poètes I", La Presse, Montréal, 10 avril 1971, p. D3.

BEAUCHAMP, Germain, "Dépossession et vie ", Le Devoir, Montréal, 21 avril 1971, p. 8.

THÉBERGE, Jean-Yves, *Gilbert Langevin, 2 fois ", Le Canada français, Saint-Jean d'Iberville, 12 mai 1971, p. 68.

PARADIS, Suzanne, "Langevin et Saint-Pierre. Demeurant à l'abri de toute définition ", Le Soleil, Québec, 31 juillet 1971, p. 34.

ANONYME, [sans titre], Le Livre canadien, Montréal, vol. II, $\mathrm{n}^{\circ} 216,1971$, s.p.

MARCOTTE, Gilles, "La poésie - Pour l'âme ", Études françaises, Montréal, vol. VIII, no 1 , février 1972, p. 94-95.

DIONNE, René, “Sur les voies de notre poésie - IV •, Relations, Montréal, n 371 , mai 1972 , p. $157-158$.

MAILHOT, Laurent, [sans titre], Livres et auteurs québécois 1971, Montréal, Éditions Jumonville, 1972, p. 138-139.

\section{Origines 1959-1967}

THÉBERGE, Jean-Yves, "Les Origines de Gilbert Langevin ", Le Canada français, Saint-Jean d'Iberville, 16 février 1972, p. 36.

DIONNE, René, "Sur les voies de notre poésie - IV *, Relations, Montréal, no 371 , mai 1972, p. 157-158.

MARCOTTE, Gilles, "La poésie - Le poète et ses mots ", Études françaises, Montréal, vol. IX, no 1 , février 1973, p. 80.

ANONYME, [sans titre], Le Livre canadien, Montréal, vol. IV, $\mathrm{n}^{\circ}$ 87, 1973, s.p.

MAILHOT, Laurent, [sans titre], Livres et auteurs québécois 1971, Montréal, Éditions Jumonville, 1972, p. 137-138.

\section{Les écrits de Zéro Legel}

PIAZZA, François, "Gilbert Langevin: un parfum de soufre ", Montréal-Matin, Montréal, 28 janvier 1973, p. 18.

MARTEL, Réginald, "Gilbert Langevin •, La Presse, Montréal, 3 février 1973, p. C3.

ANONYME, [sans titre], Le Livre canadien, Montréal, vol. IV, $\mathrm{n}^{\circ} 154$, mai 1973, s.p.

MARCOTTE, Gilles, "Les mots comme des choses... ". Études françaises, Montréal, vol. X, $\mathrm{n}^{\circ} 2$, mai 1974, p. 136. 


\section{Chansons et poèmes}

PIAZZA, François, *Un mauvais sujet de bonne compagnie!*, Montréal-Matin, Montréal, 8 juillet 1973, p. 16.

MARTEL, Réginald, [sans titre], La Presse, Montréal, 28 juillet 1973, p. D3.

\section{Novembre suivi de La vue du sang}

PIAZZA, François, "La poésie québécoise récente", Montréal-Matin, Montréal, 2 septembre 1973, p. 16.

THÉBERGE, Jean-Yves; "Lire Gilbert Langevin ", Le Canada français, Saint-Jean d'Iberville, 12 septembre 1973, p. 62.

ANONYME, "Novembre de Gilbert Langevin ", Le Canada français, Saint-Jean d'Iberville, 26 septembre 1973, p. 90.

MARCOTTE, Gilles, "Les mots comme des choses... ", Études françaises, Montréal, vol. X, n 2, mai 1974, p. 136.

\section{La douche ou la seringue}

TREMBLAY, Robert, "Gilbert Langevin ou vivre "à travers" la poésie ”, Le Soleil, Québec, 16 mars 1974, p. 74.

RICARD, François, "À propos de La douche ou la seringue. Gilbert Langevin: de zéro à la totalité „, Le Jour, Ville Saint-Laurent, 6 avril 1974, p. V3.

ANONYME, [sans titre], Le livre canadien, Montréal, vol. V, $\mathrm{n}^{\circ} 217$, juin 1974, s.p.

BROCHU, André, [sans titre], Livres et auteurs québécois 1974, Québec, Presses de l'Université Laval, 1975, p. 129-130.

\section{Chansons et poèmes 2}

DOSTIE, Gaëtan, "Lancement de Cbansons et poèmes 2. Gilbert Langevin, messager de l'événement n, Le Jour, Ville Saint-Laurent, 6 juillet 1974, p. V2.

ROYER, Jean, "Gilbert Langevin: notre espoir est un oiseau ", Le Soleil, Québec, 6 juillet 1974 , p. 38.

LEMIEUX, Jacques, "Petit bilan: Éditions Québécoises et Herbes rouges ", Le Devoir, Montréal, 31 août 1974, p. 13.

TREMBLAY, Benoît, "Gilbert Langevin : une poésie du temps qui meurt ", L'Information médicale et paramédicale, Montréal, 15 juillet 1975, p. 31.

BLAIS, Jacques, [sans titre], The University of Toronto Quarterly, Toronto, vol. XIIV, no 4, été 1975, p. 339-340.

BROCHU, André, [sans titre], Livres et auteurs québécois 1974, Québec, Presses de l'Université Laval, 1975, p. 130.

BÉDARD, Raymond, [sans titre], Nos livres, Montréal, vol. VIII, août-septembre $1977, \mathrm{n}^{\circ} 280$, s.p. 


\section{Griefs}

DE BELLEFEUILLE, Normand, "Pentes raides et terrains plats ", La Presse, Montréal, 3 avril 1976, p. D4.

HAECK, Philippe, "Poésie et griefs *, Le Devoir, Montréal, $1^{\text {er }}$ mai 1976, p. 14.

ANONYME, [sans titre], Le Livre canadien, Montréal, vol. 7, no 232, juin 1976, s.p.

ROYER, Jean, "Griefs et libertés ", Le Soleil, Québec, 5 juin 1976, p. E5.

PONTBRIAND, Jean-Noël, [sans titre], Livres et auteurs québécois 1976, Québec, Presses de l'Université Laval, 1977, p. 129-130.

\section{L'avion rose}

DE BELLEFEUILLE, Normand, "Pentes raides et terrains plats ", La Presse, Montréal, 3 avril 1976, p. D4.

BEAUSOLEIL, Claude, [sans titre], Le Jour, Ville Saint-Laurent, 9 avril 1976, p. 24.

DIONNE, René, «De la diversité en poésie québécoise •, Le Droit, Ottawa, 19 juin 1976, p. 18.

BOURASSA, André-G., "L'ange noir qu'est Langevin ", Lettres québécoises, Montréal, $n^{\circ} 3$, septembre 1976 , p. 8-10.

MAUGEY, Axel, "Attention à la poésie ", Vie des arts, Montréal, vol. XXI, nº 86, printemps 1977, p. 61.

PONTBRIAND, Jean-Noël, [sans titre], Livres et auteurs québécois 1976, Québec, Presses de l'Université Laval, 1977, p. 129-130.

\section{Mon refuge est un volcan}

GAULIN, André, [sans titre], Québec français, Québec, nº 30, mai 1978, p. 9-10.

PARADIS, Suzanne, "Langevin: le volcan n'est pas éteint ", Le Devoir, Montréal, 2 septembre 1978, p. 16.

GIGUÈRE, Richard, [sans titre], The University of Toronto Quarterly, Toronto, vol. XLVIII, n 4, été 1979, p. 360-361.

NEPVEU, Pierre, [sans titre], Livres et auteurs québécois 1978, Québec, Presses de l'Université Laval, 1979, p. 134-136.

\section{Le fou solidaire}

ALMÉRAS, Diane, [sans titre], Relations, Montréal, n 466, janvier 1981, p. 27-28.

BEAULIEU, Michel, "Gilbert Langevin. Poète visionnaire, il invente le présent ", Le Livre d'ici, Montréal, no 18, 4 février 1981, s.p.

BEAUSOLEIL, Claude, "L'amoureuse émeute de Gilbert Langevin ", Le Devoir, Montréal, 7 février 1981, p. 20 (repris sous le titre "Gilbert Langevin, issues de secours " dans Claude Beausoleil, Les livres parlent, Trois-Rivières, Écrits des Forges, 1984, p. 146-148).

BAYARD, Caroline, [sans titre], The University of Toronto Quarterly, Toronto, vol. L, $\mathrm{n}^{\circ} 4$, été 1981, p. 47. 
D'ALFONSO, Antonio, [sans titre], Nos livres, Montréal, vol. XIII, $\mathrm{n}^{\circ} 31$, janvier 1982, s.p.

BROCHU, André, "Rétrospectives et prospectives ", Voix et Images, Montréal, vol. VII, $\mathrm{n}^{\circ} 3$, printemps 1982, p. 589 (repris dans André Brochu, Tableau du poème. La poésie québécoise des années quatre-vingt, Montréal, XYZ éditeur, coll. "Documents *, 1994, p. 101-102).

CLÉMENT, Michel, [sans titre], Livres et auteurs québécois 1981, Québec, Presses de l'Université Laval, 1982, p. 99-100.

ROGERS, David F., "Pœtry chronicle ", Canadian Literature, Vancouver, nº 97 , été 1983, p. 157.

\section{Issue de secours}

POZIER, Bernard, "Issue de secours. Une tendre révolte ", Le Nouvelliste, TroisRivières, 21 novembre 1981, p. 19.

DIONNE, André, [sans titre], Nos livres, Montréal, vol. XII, nº 503, décembre 1981, s.p.

BEAUSOLEIL, Claude, "Gilbert Langevin et Gatien Lapointe ", Le Devoir, Montréal, 12 décembre 1981 , p. 39.

BROCHU, André, "Rétrospectives et prospectives ", Voix et Images, Montréal, vol. VII, $\mathrm{n}^{\circ}$ 3, printemps 1982, p. 589 (repris dans André Brochu, Tableau du poème. La poésie québécoise des années quatre-vingt, Montréal, XYZ éditeur, coll. "Documents", 1994, p. 101-102).

BRETON, Gaétan, [sans titre], Livres et auteurs québécois 1981, Québec, Presses de l'Université Laval, 1982, p. 97-98.

VISWANATHAN, Jacqueline, - La langue poétique ", Canadian Literature, Vancouver, $n^{\circ} 98$, automne 1983, p. 108.

\section{Les mains libres}

POZIER, Bernard, [sans titre], Estuaire, Montréal, no 31, printemps 1984, p. 73.

COPPENS, Patrick, "Les mains libres. Le pardon "sans pitié" d'un "pacifiste en colère" ", Mobius, Montréal, nº 21, printemps 1984, p. 95-96.

CHAMBERLAND, Roger, [sans titre], Québec français, Québec, no 54, mai 1984, p. 9.

YERGEAU, Robert, "La poésie dans tous ses états: Langevin, Vanier, Geoffroy, Van Schendel, Cloutier ", Lettres québécoises, Montréal, no 34, été 1984, p. 30-31.

BROCHU, André, "Un peu de tout ", Voix et Images, Montréal, vol. X, $\mathrm{n}^{\circ} 2$, hiver 1985, p. 189-190 (repris dans André Brochu, Tableau du poème. La poésie québécoise des années quatre-vingt, Montréal, $\mathrm{XYZ}$ éditeur, coll. • Documents", 1994, p. 102-103).

\section{Entre l'inerte et les clameurs}

ROYER, Jean, " "Les narratifs d'un monde enchevêtré " ", Le Devoir, Montréal, 8 juin 1985 ; p. 23. 
BROCHU, André, "De Gilles Hénault à King Kong ", Voix et Images, Montréal, vol. $\mathrm{XI}, \mathrm{n}^{\circ} 1$, automne 1985, p. 129-130 (repris dans André Brochu, Tableau du poème. La poésie québécoise des années quatre-vingt, Montréal, XYZ éditeur, coll. "Documents", 1994, p. 103).

GIGUÈRE, Richard, “Godbout, Hénault, Langevin: une rétrospective, deux nouveaux titres ", Lettres québécotses, Montréal, no 40, hiver 1985-1986, p. 32.

LESSARD, Alain, [sans titre], Nuit blanche, Québec, nº 22, février-avril 1986, p. 11.

\section{Comme un lexique des abîmes}

ROYER, Jean, "Le poète et son personnage *, Le Devoir, Montréal, 31 mai 1986, p. C4.

DE GONZAGUE, Louise, [sans titre], Nos livres, Montréal, vol. XVII, n 6620, juinjuillet 1986, p. 35-36.

POPOVIC, Pierre, [sans titre], Spirale, Montréal, $n^{\circ} 62$, été 1986, p. 9.

BROCHU, André, "La fiction du réel/le réel de la fiction *, Voix et Images, Montréal, vol. XII, $\mathrm{n}^{\circ}$ 2, hiver 1987, p. 329-330 (repris dans André Brochu, Tableau du poème. La poésie québécoise des années quatre-vingt, Montréal, XYZ éditeur, coll. "Documents ", 1994, p. 104).

YERGEAU, Robert, "Songe, révolte, manifeste ", Lettres québécoises, Montréal, $\mathrm{n}^{\circ} 45$, printemps 1987 , p. 38-39.

MAY, Cedric, "Entendre vibrer ", Canadian Literature, Vancouver, n ${ }^{\circ} 120$, printemps 1989, p. 201-202.

\section{Au plaisir}

D'ALFONSO, Antonio, [sans titre], Nos livres, Montréal, vol. XIX, n 3, avril 1988, p. 32.

BROCHU, André, "La critique est faillible •, Voix et Images, Montréal, vol. XIV, $\mathrm{n}^{\circ}$ 3, printemps 1989, p. 520 (repris dans André Brochu, Tableau du poème. La poésie québécoise des années quatre-vingt, Montréal, XYZ éditeur, coll. "Documents ", 1994, p. 104-105).

\section{La saison hantée}

BROCHU, André, "La critique est faillible •, Voix et Images, Montréal, vol. XIV, $\mathrm{n}^{\mathrm{O}} 3$, printemps 1989, p. 520 (repris dans André Brochu, Tableau du poème. La poésie québécoise des années quatre-vingt, Montréal, XYZ éditeur, coll. "Documents", 1994, p. 104-105).

DUMONT, François, [sans titre], Nuit blanche, Québec, $\mathrm{n}^{\circ} 36$, juin-août 1989 , p. 15.

\section{Né en avril}

ROYER, Jean, [sans titre], Le Devoir, Montréal, 28 octobre 1989, p. D3.

BROCHU, André, "Continuer et espérer *, Voix et Images, Montréal, vol. XV, $\mathrm{n}^{\circ} 3$, printemps 1990, p. $459-460$ (repris dans André Brochu, Tableau du poème. La 
poésie québécoise des années quatre-vingt, Montréal, $\mathrm{XYZ}$ éditeur, coll. "Documents ", 1994, p. 104-105).

OLSCAMP, Marcel, [sans titre], La poésie au Québec. Revue critique 1989, TroisRivières/Joliette, Écrits des Forges/Cégep Joliette-De Lanaudière, 1990, p. 6970.

\section{Haut risque}

TOUPIN, Gilles, "Oh! les beaux textes ", La Presse, Montréal, 18 novembre 1990, p. C7.

GOUGEON, Michel, [sans titre], La poésie au Québec. Revue critique 1990, TroisRivières/Joliette, Écrits des Forges/Cégep Joliette-De Lanaudière, 1991, p. 8586.

\section{Les vulnérables}

FRENETTE, Christiane, [sans titre], La poésie au Québec. Revue critique 1990, Trois-Rivières/Joliette, Écrits des Forges/Cégep Joliette-De Lanaudière, 1991, p. 153-154.

\section{Le dernier nom de la terre}

DUMONT, François, "L'art du "mais". Un ensemble de poèmes animé par un balancier ", Le Devoir, Montréal, 14 février 1993, p. D4.

CLOUTIER, Guy, [sans titre], Le Soleil, Québec, $1^{\mathrm{er}}$ mars 1993, p. B5.

CORRIVEAU, Hugues, "Drogue dure: attention poésie", Lettres québécoises, Montréal, $\mathrm{n}^{\circ} 70$, été 1993, p. 38.

PAUL, Raymond, [sans titre], La poésie au Québec. Revue critique 1992, TroisRivières/Joliette, Écrits des Forges/Cégep Joliette-De Lanaudière, 1993, p. 7677.

\section{Confidences aux gens de l'archipel}

LAURIN, Danielle, "Voix de faits ", Voir, Montréal, 16-22 septembre 1993, p. 27.

BLANCHET, Marc, [sans titre], Lectures, Montréal, octobre 1993, p. 11.

POZIER, Bernard, [sans titre], Estuaire, Montréal, n 72, janvier 1994, p. 66-68.

\section{Le cercle ouvert suivi de Hors les murs, Chemin fragile et L'eau souterraine}

LAURIN, Danielle, "Voix de faits *, Voir, Montréal, 16-22 septembre 1993, p. 27.

BLANCHET, Marc, [sans titre], Lectures, Montréal, octobre 1993, p. 13.

POZIER, Bernard, [sans titre], Estuaire, Montréal, no 72, janvier 1994, p. 63-66.

LANDRY, Gabriel, [sans titre], La poésie au Québec. Revue critique 1993, TroisRivières/Joliette, Écrits des Forges/Cégep Joliette-De Lanaudière, 1996, p. 6870. 


\section{C) Spectacles et disques}

PERREAULT, Luc, * Il ne manquait qu'un bénitier à la sortie! *, La Presse, Montréal, 6 mars 1968, p. 67.

SIROIS, André, "Gala Option-Québec. 8,000 personnes acclament leurs poètesprophètes ", Montréal, Le Devoir, 23 avril 1968, p. 2.

MAHEU, Pierre, "On est beaux, stie!", Parti pris, Montréal, vol. V, n ${ }^{\text {os }} 8-9$, été 1968, p. 74.

ROYER, Jean, "Le Québec part pour la France. La Rochelle, Paris, Avignon", Le Devoir, Montréal, 21 juin 1980, p. 19-20 là propos des Sept paroles du Québec, dont celle de G.L.].

ROYER, Jean, "Langevin, Gauvreau: deux poètes dans la ville ", Le Devoir, Montréal, 16 janvier 1981, p. 9.

ROYER, Jean, "Gilbert Langevin: cette voix que j'ai ", Le Devoir, Montréal, 2 octobre 1982, p. 35.

ROYER, Jean, "Avec Denise Boucher et Gilbert Langevin. Une contestation toujours actuelle ", Le Devoir, Montréal, 12 décembre 1984, p. 7.

LAFORGE, Christiane, "Nouvelle maison de production. Des poèmes de Langevin mis en musique [par Dominique Tremblay] •, Progrès-Dimanche, Chicoutimi, 27 octobre 1996, p. B3.

MARSOLAIS, Patrick, “ À la vie, à la mort. Dominique Tremblay et Gilbert Langevin ", Paroles et musique, Montréal, vol. IV, n 3, mars 1997, p. 7.

\section{D) Divers}

ROBERT, Guy, "Notre poésie actuelle. Troisième tranche de l'enquête de Guy Robert •, Le Devoir, Montréal, 3 février 1962, p. 11 [à propos des Éditions Atys].

BEAUliEU, Michel, *Faisceaux de la poésie québécoise ", Presqu'Amérique, Québec, vol. I, no 2, novembre-décembre 1971, p. 24 là propos de la place de G.L. dans la poésie québécoise].

RABY, Georges, "Quatre poètes de la rue, de la taverne, du quotidien " (Robert Lalonde, G.L., Pierre Léger et Michel Bujold), Perspectives-Dimancbe, Montréal, 12 décembre 1971, p. 34-38.

DUHAIME, Gérard, "Comprendre Langevin par ses souvenirs ", Progrès-Dimanche, Chicoutimi, 14 septembre 1975, p. 56 là propos de *Gilbert Langevin et le Lac-Saint-Jean ", 1975].

DEPATIE, Pierre, "La rue Saint-Denis de Roquebrune à Langevin (1900-1978) ", Village Saint-Denis, Montréal, vol. I, $\mathrm{n}^{\circ} 1$, octobre 1978, p. 11-14.

MAILHOT, Laurent et Pierre NEPVEU, La poésie québécoise. Des origines à nos jours, anthologie, Montréal, l'Hexagone, 1981, p. 35, 43 et 498; La poésie québécoise. Des origines à nos jours, nouvelle édition entièrement revue et mise à jour, Montréal, l'Hexagone, coll. "Typo", 1986, p. 24, 32 et 430 là propos de la place de G.L. dans la poésie québécoise]. 
DE GRANDPRÉ, Pierre, "Le spleen de cette génération", L'Incunable, Montréal, $18^{\mathrm{e}}$ année, $\mathrm{n}^{\circ} 1$, mars 1984, p. 23-24 [à propos du fonds Gilbert-Langevin à la Bibliothèque nationale du Québec].

ROYER, Jean, Introduction à la poésie québécotse. Les poètes et les cuvres des origines à nos jours, Montréal, Leméac, coll. « BQ *, 1989, p. 93 [à propos de la place de G.L. dans la poésie québécoise].

GAUVIN, Lise et Gaston MIRON, Écrivains contemporains du Québec depuis 1950, Paris, Seghers, 1989, p. 323 là propos de la place de G.L. dans la poésie québécoise].

BAILLARGEON, Normand, “Chronique à quatre mains ", Le Devoir, Montréal, 2 décembre 1996, p. A6 lanalyse de l'actualité à partir d'A Ange-animal ", chanson de G.L.].

\section{TÉMOIGNAGES ET HOMMAGES}

DOR, Georges, Si tu savais..., essai autobiographique suivi des paroles et partitions de dix-neuf chansons, préface de Gaston Miron, Montréal, Éditions de l'Homme, 1977, p. 45-49.

BRUNET, Yves-Gabriel, • En finira-t-il un jour de naître... •, dans Gilbert Langevin, Au plaisir, Trois-Rivières, Écrits des Forges, coll. “Radar ", n 25, [septembre] $1987,4^{\mathrm{e}}$ de couverture.

MIRON, Gaston, ، En guise de postface. Témoignage présenté lors d'une soirée en hommage à l'auteur, au Salon du livre du Saguenay-Lac-Saint-Jean * (30 septembre 1988), dans Gilbert Langevin, Né en avril, Trois-Rivières, Écrits des Forges, coll. * Radar ", no 37, 1989, p. 57-59.

BAILLARGEON, Normand, "Mon beau rossignol ", Le Devoir, Montréal, 15 août 1995, p. A6 (repris partiellement dans PoéVie, 1997).

LÉVESQUE, Robert, "Le poète Gilbert Langevin n'est plus *, Le Devoir, Montréal, 19 octobre 1995, p. A5.

CASSIVI, Marc et Mario ROY, "Mort d'un poète itinérant: Gilbert Langevin (19381995)., La Presse, Montréal, 19 octobre 1995, p. D7.

VILLENEUVE, Paul, "Décès du poète Gilbert Langevin *, Le Journal de Montréal, Montréal, 19 octobre 1995, p. 52.

ROYER, Jean, "Gilbert Langevin: la leçon de la vérité des mots ", Le Devoir, Montréal, 21 octobre 1995, p. D8 (repris dans le Bulletin UNEQ, Montréal, hiver 1996 [février 1996], p. 3).

MARTEL, Réginald, "Gilbert Langevin: de la souveraineté de l'amour ", La Presse, Montréal, 22 octobre 1995, p. B3.

BRUNET, Yves-Gabriel, «Hommage posthume à Gilbert Langevin • (prononcé lors des funérailles, 21 octobre 1995), La Presse, Montréal, 31 octobre 1995, p. B2.

LACHANCE, André, "Va, le vagabond de l'absolu... ", La Presse, Montréal, 5 novembre $1995, \mathrm{p}$. B2.

DOSTIE, Gaëtan, "Gilbert Langevin 1938-1995 — L'ultime rencontre ", Bulletin UNEQ, Montréal, hiver 1996 [février 1996], p. 2. [Ce témoignage propose une 
transcription du dernier écrit de Gilbert Langevin, fait en présence de l'auteur à l'hôpital Notre-Dame, le 13 octobre 1995.]

POZIER, Bernard, ·Je me souviens... ", Bulletin UNEQ, Montréal, hiver 1996 [février 1996], p. 3.

CORRIVEAU, Hugues, "Gilbert Langevin, le fou solidaire", Lettres québécoises, Montréal, $n^{\circ} 81$, printemps 1996, p. 9.

BRUNET, Yves-Gabriel, "Le secret se cache dans le cœur des fous..." Gilbert Langevin ", dans Hélène Tremblay (voix) et Dominique Tremblay (violon), Langevin/Tremblay/Fous solidaires, CD, Val-Morin, SRM, SRM 02502, [mai] 1996. 